\title{
Successful Restoration of Complete Heart Block to Normal Sinus Rhythm by Primary Angioplasty of Dual Left Anterior Descending Artery
}

\author{
Santosh Kumar Sinha ${ }^{a}$,, Vikas Mishra ${ }^{a}$, Mukesh Jitendra Jha ${ }^{a}$, Mahmadula Razi ${ }^{a}$, Nasar Abdalia, \\ Anupam Mahrotra ${ }^{a}$, Mohammad Asif ${ }^{a}$, Lokendra Rekwal ${ }^{a}$, Vikas Chaturvedia, \\ Shravan Singh ${ }^{a}$, Vinay Krishna ${ }^{a}$
}

\begin{abstract}
Dual left anterior descending (LAD) artery is a rare coronary anomaly. We present a patient with a rare case of dual LAD, smaller one arising from the left main coronary stem and larger one from right coronary artery who presented with acute anterior wall myocardial infarction with complete heart block (CHB). Temporary pacemaker was implanted and coronary angiogram revealed critical occlusion of proximal LAD which was subsequently revascularized by primary angioplasty using drug-eluting stent (Xience prime, $2.75 \times 23 \mathrm{~mm}$ ) leading to recovery of $\mathrm{CHB}$ and restoration to normal rhythm. To the best of our knowledge, this is the first reported case of dual LAD presenting with $\mathrm{CHB}$ treated by primary angioplasty reported in the literature.
\end{abstract}

Keywords: Dual left anterior descending artery; Complete heart block; Myocardial infarction; Temporary pacemaker; Primary angioplasty

\section{Introduction}

In the vast majority of people, the left anterior descending (LAD) coronary artery branches out of the left main coronary artery (LMCA) shaft and courses along the anterior interventricular groove (AIVG) all the way toward the apex of the heart. The presence of two LAD coronary arteries, referred to as the dual LAD anomaly, is a rare congenital anomaly [1]. Spindola-Franco et al [2] have initially described and characterized the first four types of this anomaly based on the origin and course of both a short and a long branch of the LAD. Since then, with the increase in the number of invasive coronary angiographies, and more importantly with the advent of coronary computed tomography, new types have been further described.

Manuscript accepted for publication March 14, 2017

aDepartment of Cardiology, LPS Institute of Cardiology, G.S.V.M. Medical College, Kanpur, Uttar Pradesh 208002, India

${ }^{b}$ Corresponding Author: Santosh Kumar Sinha, Department of Cardiology, LPS Institute of Cardiology, G.S.V.M. Medical College, Kanpur, Uttar Pradesh 208002, India. Email: fionasan@rediffmail.com

doi: https://doi.org/10.14740/cr532w
Most of the identifications were performed in lieu of evaluation of either atypical chest pain or angina. Recognition at coronary imaging is critical as both surgical and percutaneous reperfusion strategies may be altered by its presence [2].

\section{Case Report}

A 42-year-old male patient with a history of diabetes mellitus and dyslipidemia presented with excruciating retrosternal chest pain and sweating of $1 \mathrm{~h}$ duration. His physical examination and biochemistry were all unremarkable. Electrocardiogram revealed ST-elevation in $\mathrm{V}_{1}-\mathrm{V}_{4}$ with complete heart block and reciprocal changes in inferior leads. There was mild hypokinesia in left anterior descending territory (LAD Tx) with ejection fraction of $48 \%$. He was taken for primary percutaneous coronary intervention (PCI) through radial route after proper consent. He was preloaded with prsugrel $60 \mathrm{mg}$, aspirin $325 \mathrm{mg}$ and atorvastatin $80 \mathrm{mg}$. Temporary pacing lead was put through right jugular vein. Radial artery was cannulated by $21 \mathrm{G}$ needle and 0.021 " guidewires (Avanti transradial kit; Cordis Corp., USA) were inserted. A 6 F sheath was put and cocktail containing $200 \mu \mathrm{g}$ nitroglycerin, $2.5 \mathrm{mg}$ diltiazem, and 2,500 IU unfractioned heparin was injected. Angiogram was performed by $5 \mathrm{~F}$ TIG catheter (Terumo, Japan). Angiogram of left system revealed near total occlusion of proximal LAD artery with normal flowing non-dominant circumflex artery (Fig. 1). Right coronary artery was dominant, disease-free and giving large LAD artery (Fig. 2). LAD was cannulated with EBU 3.5 6 F (Launcher, Medtronic, USA) and 5,000 IU unfractioned heparin was further injected. A BMW guidewire 0.014 ", $140 \mathrm{~cm}$ (Abott, USA) was parked beyond the lesion (Fig. 3). Lesion was pre-dilated with $2 \times 10 \mathrm{~mm}$ Minitrak balloon (Abott, USA) to $12 \mathrm{~atm}$ (Fig. 3) and was stented by deploying $2.75 \times 23 \mathrm{~mm}$ Xience prime stent (Everolimus eluting stent, Abott, USA) up to 13 atm pressure (Fig. 4) achieving TIMI III flow in LAD (Fig. 5a, b). His symptom stabilized and ECG reverted back to normal sinus rhythm. Pacing wire was removed next day. Multi-detector coronary computed tomography (MDCT) with volume rendered reconstruction was done to delineate the course of LAD which revealed larger LAD (l-LAD) was coursing in front of pulmonary trunk to reach anterior interventricular groove (Fig. 6a, b). Based on findings of invasive angiogram and MDCT, diagnosis of dual LAD type 


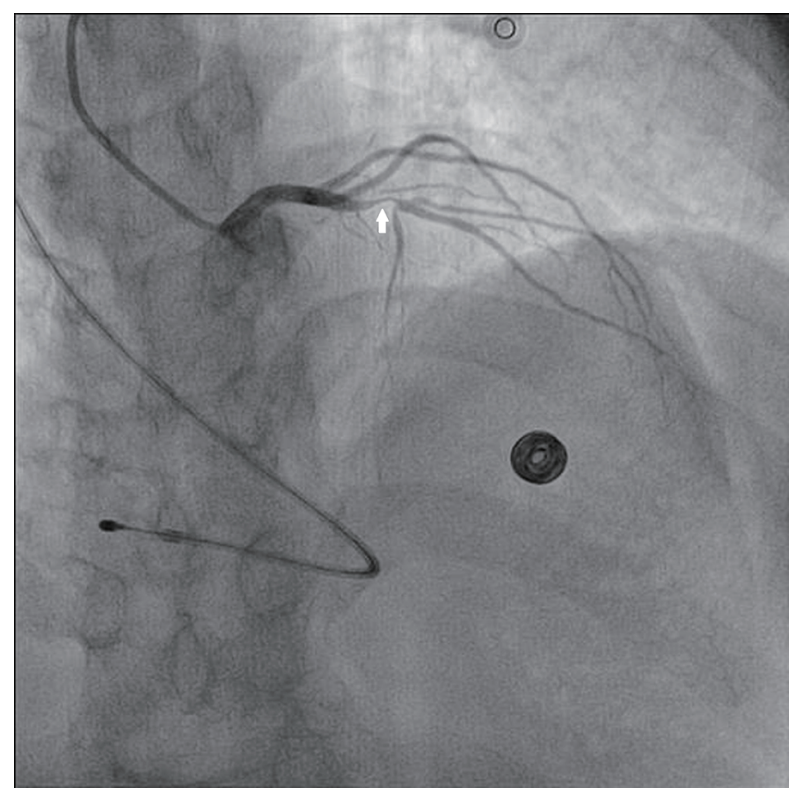

Figure 1. Near total occlusion of proximal small LAD (s-LAD) by white arrow in antero-posterior (AP) caudal view.

4 was made. The patient was discharged on the following day with aspirin $150 \mathrm{mg} /$ day, prasugrel $10 \mathrm{mg} /$ day, atorvastatin 80 $\mathrm{mg}$ /day and ramipril $2.5 \mathrm{mg} /$ day. Patient is doing excellent since then with regular follow-up at our institute.

\section{Discussion}

Dual LAD circulation has been reported to occur with an incidence of around $1 \%$ on invasive coronary angiography [2].

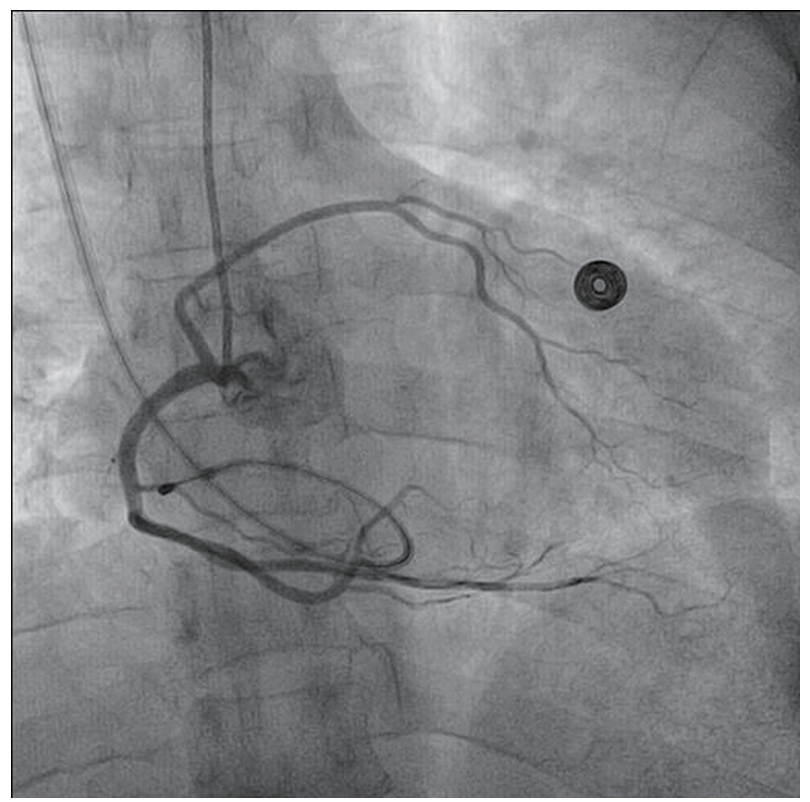

Figure 2. RCA dominance giving rise to $L A D$ in left anterior oblique (LAO) view with temporary lead in situ.

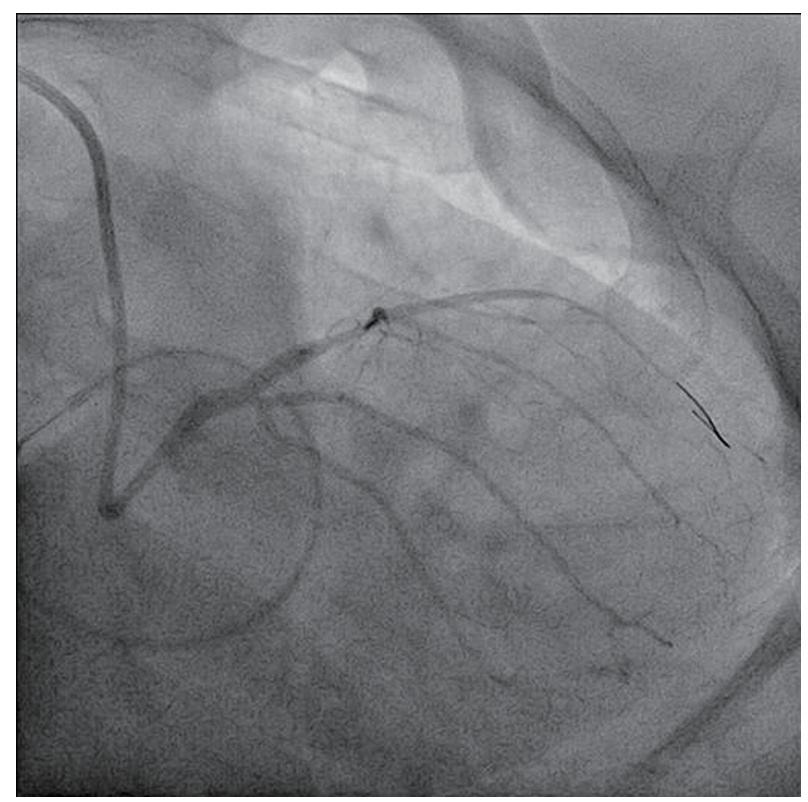

Figure 3. BMW guidewire being parked beyond the lesion in AP caudal view with pre-dilatation with $2 \times 10 \mathrm{~mm}$ Minitrak balloon.

Type 1 is the most prevalent and constituted more than $85 \%$ of the cases as described by Bozlar et al [3], characterized by one short and one long LAD which both branch off the LMCA and supply the proximal and distal AIVG, respectively. The other types are relatively rare and up to nine types have been described [3]. Type 4 is described as one where proper LAD is absent, short LAD arises from left main trunk to terminate into proximal anterior interventricular septum (AIS) and long LAD originates from proximal right coronary artery, follows an anomalous prepulmonic course anterior to the right ven-

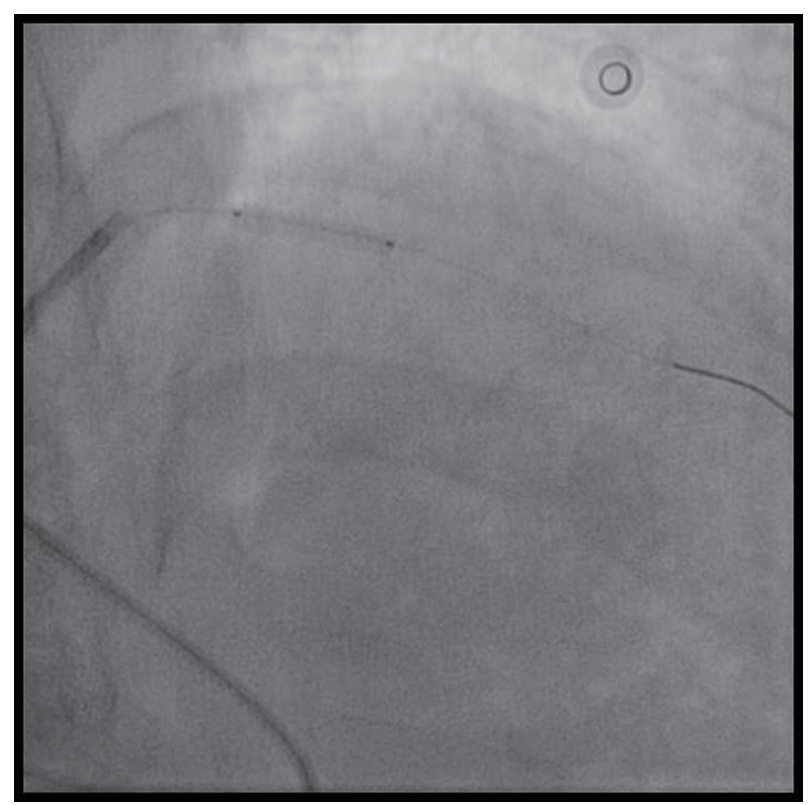

Figure 4. Lesion being stented by deploying $2.75 \times 23 \mathrm{~mm}$ Xience prime drug-eluting stent. 


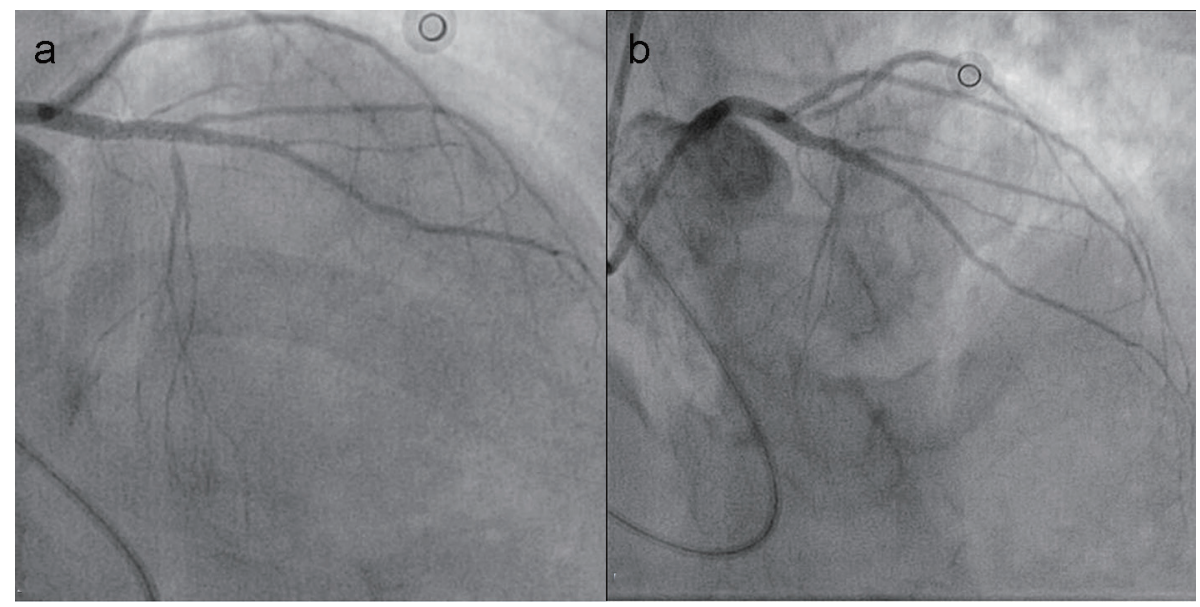

Figure 5. Post PCI TIMI III flow of culprit artery in AP caudal view (a) and AP cranial view (b).

tricular outflow tract, and enters the distal AIS. Our case was type 4 with few unique differences: first, shorter one was not terminating into proximal AIS; second, longer was entering into proximal AIS instead of distal; third, it was shorter one responsible for causing acute coronary syndrome meaning to say that though anatomically short, it may be functionally and physiologically as important as long LAD. What was even more interesting is that our case manifested with CHB which has not been reported with any types of dual LAD till now. With the complete revascularization, most symptoms and electrocardiogram reverted. CHB though portends grave prognosis if associated with anterior wall myocardial infarction because of amount of myocardium at jeopardy, if successfully revascularized within golden hour, outcome can be improved. Also, acute coronary syndrome treated by primary PCI is also being reported for first time as it is thought that dual LAD circulation carries a benign course. Identifying specific coronary artery anomalies and characterizing their respective anatomy are usu- ally difficult with catheter coronary angiography alone due to the unavailability of spatial information regarding the origin and course of the coronary arteries in relation to the different heart structures. This becomes a critical issue whenever an anomalous artery courses in between the aorta and the right ventricular outflow tract as in type 6 . It is similar to the previously described type 4 except it courses in between the right ventricular outflow tract and the aortic root [4]. This course taken by the long LAD in the type 6 will put the vessel at risk of compression with blood flow compromise and resultant sudden cardiac death in situations of increased pulmonary blood flow [5]. Therefore, cardiac computed tomography angiography is essential whenever dual LAD anomaly is discovered on invasive coronary angiography since it confers adequate comprehension of the relationship of the coronary artery tree to other heart structures and thus helps in risk stratification. Within this same context, being both aware and familiar with this anomaly is extremely essential for the adequate planning

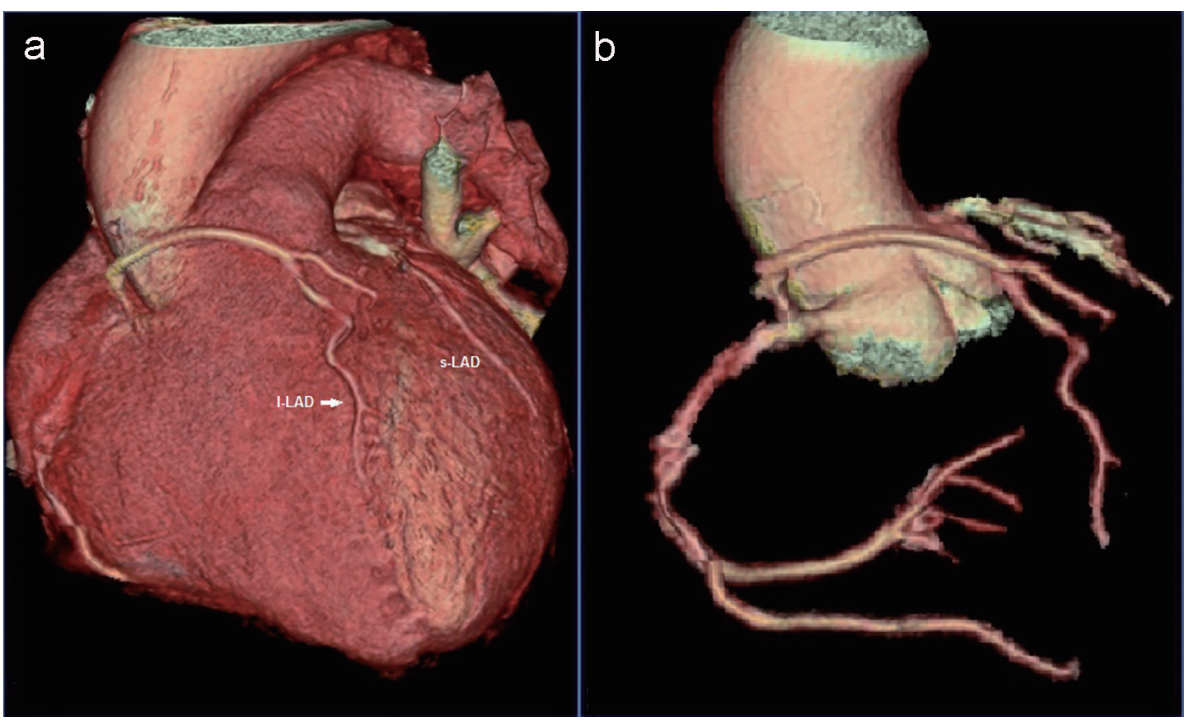

Figure 6. 3D volume-rendered $C T$ angiographic reconstruction showing LAD coursing in front of pulmonary trunk (a) and MDCT showing RCA giving rise to LAD (b). 
and management of associated coronary artery disease, whether it was through PCIs or coronary artery bypass grafting.

\section{Acknowledgments}

We thank Vinay Singh, Ritesh and Risabh for their technical support in performing and interpreting MDCT.

\section{Funding}

None.

\section{Conflicts of Interest}

None.

\section{References}

1. Agarwal PP, Kazerooni EA. Dual left anterior descending coronary artery: CT findings. AJR Am J Roentgenol. 2008;191(6):1698-1701.

2. Spindola-Franco H, Grose R, Solomon N. Dual left anterior descending coronary artery: angiographic description of important variants and surgical implications. Am Heart J. 1983;105(3):445-455.

3. Bozlar U, Ugurel MS, Sari S, Akgun V, Ors F, Tasar M. Prevalence of dual left anterior descending artery variations in CT angiography. Diagn Interv Radiol. 2015;21(1):34-41.

4. Maroney J, Klein LW. Report of a new anomaly of the left anterior descending artery: type VI dual LAD. Catheter Cardiovasc Interv. 2012;80(4):626-629.

5. Patel S. Normal and anomalous anatomy of the coronary arteries. Semin Roentgenol. 2008;43(2):100-112. 